\title{
ЗНАЧЕНИЕ ЦИТОКИНОВОГО ПРОФИЛЯ ПРИ МАНИФЕСТНОМ ГИПОТИРЕОЗЕ РАЗЛИЧНОГО ГEHE3A
}

Орлова М.М., Знаменшикова Г.Н., Дихт Н.И.

\author{
ФГБОУ ВО «Саратовский ГМУ им. В.И. Разумовского »Минздрава России, Саратов
}

Аутоиммунный тиреоидит сопровождается лимфоцитарной инфильтрацией щитовидной железы, синтезом антител к тиреопероксидазе и дисбалансом синтеза цитокинов. Представляет интерес изучение особенностей синтеза про- и противовоспалительных цитокинов в условиях развития воспалительных и аутоиммунных процессов и их динамика в процессе лечения.

ЦЕЛЬ: исследование уровня цитокинов у пациентов с манифестным гипотиреозом различного генеза в стадии декомпенсации и на фоне лечения.

МАТЕРИАЛЫ И МЕТОДЫ: проводилось исследование уровней цитокинов у 52 пациентов с первичным манифестным гипотиреозом в возрасте от 32 до 50 лет: 23 пациента с послеоперационным гипотиреозом и 29 пациентов с аутоиммунным тиреоидитом (АИТ). Обследовано 8 мужчин (15\%) и 44 женщины (85\%). В контрольную группу было включено 20 практически здоровых лиц. Уровни интерлейкина-1 $\beta$ (ИЛ-1ß), интерлейкина-6 (ИЛ-6), интерлейкина-8 (ИЛ-8), ИФН-ү, интерлейкина-10 (ИЛ-10) определяли методом твердофазного иммуноферментного анализа (тест-системы ЗАО «Вектор-Бест»).

РЕЗУЛЬТАТЫ: согласно полученным результатам исследований, на фоне метаболических нарушений, связанных со снижением уровня основного обмена и угнетением активности защитных систем, выявлены значимые изменения некоторых цитокинов. При манифестном гипотиреозе наблюдается дисбаланс прои противовоспалительных цитокинов с преобладанием активности провоспалительного звена: уровни интерлейкина-6, интерлейкина-8, ИФН-ү значимо повышены, уровень интерлейкина-10 (ИЛ-10) остается в пределах референса, но значимо выше уровня средних значений контрольной группы.

Выявлена статистически значимая прямая корреляционная зависимость между уровнем ТТГ в сыворотке крови и уровнем ИФН-ү в группе пациентов с АИТ, что, вероятно, обусловлено выраженностью воспалительной реакции в условиях развития аутоиммунного процесса и апоптоза тимоцитов. Содержание ИЛ-8 у пациентов с АИТ значимо превышает соответствующий показатель в контрольной группе $13,3[8,8 ; 14,6]$ vs $5,48[5,2 ; 6,9]$ пг/мл $(p(U)=0,03)$. В группе послеоперационного гипотиреоза только уровень ИЛ-8 значимо повышен в сравнении в контролем, однако, при сравнении уровней ИЛ-8 в группах АИТ и послеоперационного гипотиреоза значимых отличий не выявлено. Полученные результаты позволяют предполагать, что данный хемокин принимает активное участие в механизме начального этапа аутоиммунного процесса, направляя антигензависимые лимфоциты, которые секретируют спектр провоспалительных цитокинов. Результаты исследования уровня ИЛ-1ß в группе пациентов с ХАИТ и ПГ соответствовали диапазону нормальных значений.

\section{выводы.}

1. В группе пациентов с АИТ выявлена несбалансированная гиперцитокинемия с увеличением уровня как провоспалительных (ИЛ-6, ИЛ-8), так и противовоспалительных (ИЛ-10) цитокинов.

2. Установлена взаимосвязь между гиперпродукцией провоспалительных цитокинов ИЛ-6, ИЛ-8 и уровнем ТТГ с наибольшей степенью выраженности у пациентов с аутоиммунным тиреоидитом.

КЛЮЧЕВЫЕ СЛОВА: гипотиреоз, тиреоидные гормоны, цитокины, аутоиммунный тиреоидит. 